\title{
Impact of Vaginal Antifungal Products on Utilization of Health Care Services: Evidence from Physician Visits
}

\author{
Martin S. Lipsky, MD, Teresa Waters, PbD, and Lisa K. Sharp, PbD
}

Background: Recently many medications formerly available only by prescription have been approved for over-the-counter (OTC) status. In 1990, clotrimazole became the first available OTC drug to treat candidal vaginitis. Subsequently several other prescription antifungal medications have also been available in OTC products. One proposed benefit of these switches from prescription to OTC status is a reduction in the utilization of health care services.

Metbods: Using National Ambulatory Medical Care Survey data, the average numbers of annual visits for vaginal complaints were estimated for 1985,1990 , and 1994. These years were chosen because they represented periods before, close to, and after the approval of the OTC antifungal preparations. The estimated visits for each year were compared using a chi-square analysis with a sample weight correction.

Results: There was a 15\% decline in the number of vaginitis visits from 1990 to 1994 that potentially could be attributed to the availability of the OTC antifungal preparations. The decrease in physician visits results in approximately $\$ 45$ million in direct cost savings and another $\$ 18.75$ million in indirect savings by reducing time lost from work.

Conclusions: It appears that the availability of OTC anticandidal fungal preparations reduces the number of physician visits for vaginitis, resulting in cost savings. (J Am Board Fam Pract 2000;13: 178-82.)

Over-the-counter (OTC) drugs are pharmaceutical agents directly available to the consumer for purchase. Prescription medicines are being approved for OTC status with increasing frequency. Currently more than 600 OTC preparations contain ingredients that previously were available only by prescription. ${ }^{1}$ Many recently approved drugs, such as $\mathrm{H}_{2}$-blockers, are among the most commonly used medications, with a total retail value exceeding $\$ 8$ billion. ${ }^{2}$ Despite the substantial impact OTC medications have on medical care, the trend of approving drugs for OTC status has not been extensively studied.

One potential advantage of the prescription-toOTC movement is the potential to reduce health care costs. For example, it is estimated that more than $\$ 1$ billion in health care costs were saved in the

Submitted, revised, 24 January 2000.

From the Department of Family Medicine (MSL, LKS), Northwestern University Medical School, Chicago; and the Institute for Health Services Research and Policy Studies (TW), Evanston, Ill. Address reprint requests to Martin S. Lipsky, MD, Department of Family Medicine, Northwestern University Medical School, 303 East Chicago Ave, Morton 1-658, Chicago, IL 60611. first few years after hydrocortisone was approved for nonprescription status. ${ }^{3,4}$ OTC proponents argue that these products decrease cost by reducing the use of health care services because a physician's visit is not needed to obtain an OTC medication. ${ }^{5,6}$ Fewer physician visits should result in decreased direct costs for physician fees, potentially lowering health care costs. In addition, indirect savings could result by reducing time lost from work and the travel costs associated with a health care visit.

Each year vaginitis accounts for more than 10 million physician office visits, making it one of the most common medical complaints. ${ }^{7}$ In 1990 the Food and Drug Administration approved clotrimazole, an imidazole, as the first available OTC drug to treat vaginal candidal infections. Subsequently, other antifungal medications, such as miconazole and butoconazole, have become available as OTC preparations. Considering the number of woman who suffer from vaginitis, the nonprescription availability of these agents could have an enormous influence on clinical practice. Despite this potential impact, there is little research examining the effect of the prescription- 
to-OTC movement of these products on the utilization of health care services.

A previous study examining the impact of availability of OTC antifungal preparations on physician visits in a single health maintenance organization found that physician visits for vaginitis were reduced by 0.66 visits per 100 members. ${ }^{8}$ In this study, we sought to generalize this finding using data from the National Ambulatory Medical Care Survey (NAMCS). Specifically, we sought to compare the average number of visits for vaginitis complaints before and after the availability of OTC antifungal preparations.

\section{Methods}

\section{Data Source}

NAMCS is an annual survey of US office-based physicians visits, conducted and supported by the National Center for Health Statistics since 1973. NAMCS is intended to provide information about community-based ambulatory practices. Physician participants are selected from the master files of the American Medical Association and the American Osteopathic Association. The sample excludes physicians practicing in health care centers, some hospital outpatient departments, and emergency departments. NAMCS records a physician-reported 1 -week random sample of patient visits. For each patient visit, physicians or their office staff complete encounter forms on patient demographics, patient complaints, diagnosis, procedures, and prescribed medications (including OTC medications).

\section{Visit Selection Criteria}

The NAMCS data set contains information on individual physician encounters with up to three patient-reported complaints. For the purpose of this study, visits for women aged between 15 and 64 years were selected when any of the following vaginal symptoms was listed as one of the three complaints: vaginal discharge, vaginal pain, vaginal infection, vaginal burning, or vaginitis. Three years of data were reviewed, from January 1 through December 31 for each of the years 1985, 1990, and 1994. ${ }^{9-11}$ These years were selected because they represent periods before, close to, and after the approval of the OTC antifungal preparations.

\section{Data Analysis}

The analysis incorporates two data sources: NAMCS and the Bureau of Census data. Because the NAMCS data do not take into consideration the population of women aged between 15 and 64 years during that year, data were obtained from the Bureau of Census ${ }^{12}$ to calculate population-based estimates for each year. Estimates for 1985, 1990, and 1994 were calculated for the following:

1. The average number of vaginal-related physician visits per woman (aged 15 to 64 years) per year

2. The average number of physician visits (all causes) per woman (aged 15 to 64 years) per year

3. The ratio of vaginitis-related visits to all physician visits for any complaint for the selected population

When comparing these estimates across the 3 years, it should be noted that the sample design is not perfectly suited for determining whether the differences between years are statistically significant. An ideal data set would include comprehensive health care utilization data for a random sample of women in the United States. This data set could be used to construct the rates, and simple $t$ tests could be used to explore statistical significance. NAMCS data are a random sample of office visits, not patients. For this reason, each observation must be weighted according to the number of patients that the observed visit might represent by incorporating census data. This method is commonly used in the econometric literature and is referred to as correction for choice-based sampling. For example, if the observed visits overrepresent a certain demographic group, such as older women, NAMCS provides a correction factor to account for this oversampling. Statistical significance was tested using chi-square analysis that incorporated the correction factor provided by NAMCS plus population estimates provided by census data. In essence, each observation (i) is inflated by a factor $\left(p_{y y}\right)(y y=85,90,94)$ defined as follows:

$$
\mathrm{p}_{y y}^{\mathrm{i}}=\mathrm{N}_{y y} / w \mathrm{t}_{y y}^{\mathrm{i}} \mathrm{n}_{y y}
$$

where $\mathrm{n}_{\mathrm{yy}}$ is the number of observations (visits) in year $y y, w^{i}{ }_{y y}$ is the sample weight (provided by NAMCS), and $\mathrm{N}_{\mathrm{yy}}$ is the total number of women aged 15 to 64 years. This number is significantly greater than 1 because each observation represents 
Table 1. Vaginitis Visits, Physician Office Visits, and Ratio of Vaginitis Visits to All Physician Office Visits, Women Aged 15 to 64 years: 1985, 1990, 1994.

\begin{tabular}{llll}
\hline Characteristics & 1985 & 1990 & 1994 \\
\hline $\begin{array}{l}\text { Vaginitis visits per } 1000 \text { women } \\
\text { Physician office visits per } 100 \text { women }\end{array}$ & 0.101 & 0.091 & $0.070^{*}$ \\
$\begin{array}{l}\text { Ratio of vaginitis visits to all physician } \\
\text { office visits }\end{array}$ & 0.032 & 0.029 & $0.024^{\ddagger}$ \\
\hline
\end{tabular}

Note: unless otherwise indicated, differences not significant. * 1994 rate significantly lower than $1985(\mathrm{P}<.05)$.

+1994 rate significantly lower than $1985(\mathrm{P}<.01)$ and 1990 ( $\mathrm{P}<.05)$.

$\mp 1994$ rate significantly lower than $1985(\mathrm{P}<.01)$ and 1990 $(\mathrm{P}<.01)$.

many women in the United States. This number is not the same for each observation (ie, $\mathrm{p}^{\mathrm{i}}=\mathrm{N}_{\mathrm{yy}}$ / $\mathrm{n}_{\mathrm{yy}}$ ), because we are not working with a random sample.

\section{Results}

The estimated total number of visits with a vaginitis-related diagnosis per year for women aged between 15 and 64 years was 8 million for $1985,8.2$ million for 1990, and 6 million for 1994. The total number of visits for any complaint during 1985, 1990 , and 1994 for the same population group was 249.8 million, 278.1 million, and 250.9 million, respectively. Table 1 shows the calculated estimates for number of vaginitis visits per 100 women, number of (all) visits per 100 women, and a ratio for each year. There is a nonsignificant decline between 1985 (before the availability of OTC vaginal antifungal medications) and 1990 (around the time when prescription antifungal medications were approved for OTC status) both in overall visits per 100 women and in vaginitis visits per 100 women. The decline between 1985 and 1994 (postapproval) is statistically significant for both overall visits and for vaginitis-related visits. To control for the decline in overall visits between periods relative to the decline in vaginitis-related visits, vaginitis-related visits (per 100) were divided by all visits (per 100). As Table 1 illustrates, this ratio of vaginitis visits to all visits also declined significantly during the 1985 to 1994 period, supporting the hypothesis that the decline in vaginitis visits cannot be attributed to the overall decline in office visits.

Estimating the total number of visits potentially saved by the availability of OTC antifungal prod- ucts is complicated by a decreasing number of total visits and vaginitis-related visits in the period before the antifungal product approval. In the 5-year period between 1985 to 1990 , vaginitis visits decreased $10 \%$, or approximately $2 \%$ per year. In contrast, vaginitis visits decreased $23 \%$ in the 4-year period from 1990 to 1994 . Assuming that there was a continued $2 \%$ annual baseline drift, approximately $8 \%$ of the total $23 \%$ decrease in vaginitis visits in the 1990 to 1994 period could be attributed to baseline drift. The remaining $15 \%$ ( $23 \%$ to $8 \%$ ) could be attributed to the availability of the OTC antifungal agents. This $15 \%$ decline in the number of physician visits translates into a decrease of approximately 1 million visits per year after approval for OTC status. Using a rough estimate of the cost of a physician office visit as $\$ 61,{ }^{13}$ this decrease amounts to more than $\$ 45$ million in direct cost savings per year. If one also considers the indirect costs associated with time lost from work when visiting a physician, the annual savings attributable to the vaginal anticandidal medication switch from prescription to OTC status increases by another $\$ 18.75$ million (1 million visits $\times 2$ hours $\times$ average wage of $\$ 9.37 / \mathrm{h}$ [Bureau of Labor Statistics 1994 data]).

\section{Discussion}

The study results indicate that the average number of vaginitis visits per women has decreased significantly since 1990 , supporting the contention that availability of OTC antifungal medications reduces use of health care services. Based on the NACMS data, once the anticandidal fungal preparations became available without a prescription, there was a $15 \%$ decrease of approximately 1 million office visits annually for vaginitis. The reduced number of visits supports the contention that enhanced consumer availability of the antifungal preparations has empowered women to self-medicate for vaginal symptoms. Fewer visits also suggest that health care costs were reduced by the availability of OTC antifungal agents.

It should be noted, however, that the cost savings attributable to OTC approval might not be quite so simple as the direct and indirect savings from reduced physician visits. Antifungal preparation sales have almost doubled since being approved for OTC status despite little evidence that there is a concomitant increase in the incidence of 
candidal vaginitis, strengthening the suspicion that these products might be overused, particularly in patients for whom the initial diagnoses of a candidal infection was not clearly established. ${ }^{14}$ Using these preparations inappropriately might mitigate the cost savings from reduced visits. In addition to a woman potentially treating herself needlessly, misdiagnosis could result in delaying treatment for a more serious condition, causing both increased morbidity and expense. Furthermore, external costs related to such issues as increased biologic resistance to these agents might lessen the apparent savings. Finally, the potential exists for negative consequences from the decreased number of contacts with health professionals. A more detailed analysis incorporating the multiple economic ramifications would help estimate the actual economic savings. Any economic analysis should also take into account the potential improvement in quality of life by having an immediately available treatment.

The study has several limitations that deserve mentioning. First, we looked at only one OTC product, and the findings might not necessarily mean that other prescription products approved for OTC formulation result in fewer physician visits. Antifungal preparations are not unique, however, and it is not unreasonable to suspect that other products, such as $\mathrm{H}_{2}$-blockers, will also reduce the number of physician visits as they become available in OTC preparations. ${ }^{15,16}$ Second, the decreased number of vaginal visits noted might be from confounding variables, such as changes in coding or improved technology for diagnosing vaginitis since 1985. Nevertheless, the basic criterion for diagnosing fungal vaginitis has not changed much during the past decade. ${ }^{17}$ In addition, the study examining a health maintenance organization population observed similar decreases in vaginitis visits with availability of OTC antifungal agents. ${ }^{8}$ This finding supports the likelihood that a considerable portion of the decreased number of visits is related to the availability of OTC products. Finally, the data were interpolated from NAMCS, which is physician reported and might not accurately reflect national practice patterns. Even so, previous validation studies performed in early years have confirmed the general accuracy of the information obtained.

In conclusion, it appears that the availability of OTC anticandidal fungal preparations reduces the number of visits to physicians for vaginitis. $\mathrm{Al}$ though this finding appears to result in substantial cost savings, further analysis examining economic impact is warranted. Issues regarding loss of physician contact and quality of life are also important for determining the overall value of OTC antifungal preparations.

\section{References}

1. Snyder K. Marketing consumer trends driving switch-hitters up: prescription drugs changing to over-the-counter status. Drug Topics 1957;89:141.

2. Economic benefits of self-medication. Washington, DC; Kline \& Company, 1997.

3. Temin P. Cost and benefits in switching drugs from Rx-to-OTC. J Health Econ 1983;2:187-205.

4. Morgan PP, Cohen L. Off the prescription pad and over the counter: the trend toward drug deregulation grows. CAMJ 1995;152:387-9.

5. Gossel TA. Implication of the reclassification of drugs from prescription-only to over-the-counter status. Clin Ther 1991;13:200-15.

6. Lipsky MS, Waters T. The "prescription-to-OTC switch" movement: Its effects on antifungal vaginitis preparations. Arch Fam Med 1999;8:297-300.

7. Kent HL. Epidemiology of vaginitis. Am J Obstet Gynecol 1991;165(4 Pt 2):1168-76.

8. Gurwitz JH, McLaughlin TJ, Fish LS. The effect of an $\mathrm{Rx}$-to-OTC switch on medication prescribing patterns and utilization of physician services: the case of vaginal antifungal products. Health Serv Res 1995;30:672-85.

9. National Ambulatory Medical Care Survey, 1984. Hyattsville, Md: National Center for Health Statistics, Department of Health and Human Services. Ann Arbor, Mich: Inter-university Consortium for Political and Social research [distributor], 1995.

10. National Ambulatory Medical Care Survey, 1990. Hyattsville, Md: National Center for Health Statistics, Department of Health and Human Services. Ann Arbor, Mich: Inter-university Consortium for Political and Social research [distributor], 1995.

11. National Ambulatory Medical Care Survey, 1994. Hyattsville, Md: National Center for Health Statistics, Department of Health and Human Services. Ann Arbor, Mich: Inter-university Consortium for Political and Social research [distributor], 1995.

12. National and state population estimates. Washington, DC: Bureau of the Census, Department of Commerce, Economics and Statistics Administration, 1995.

13. Source book of the health insurance data. Washington, DC: Health Insurance Association of America, 1994.

14. Nyirjesy P, Weitz MV, Grody MH, Lorber B. Over- 
the counter and alternative medicines in the treatment of chronic vaginal symptoms. Obstet Gynecol 1997;90:50-3.

15. Andrade SE, Gurwitz JH, Fish LS. The effect of an $\mathrm{Rx}$-to-OTC switch on medication prescribing patterns and utilization of physician services: the case of $\mathrm{H}_{2}$-receptor antagonists. Med Care 1999;37:424-30.
16. Oster G, Huse DM, Delea TE, Colditz GA, Richter $J M$. The risks and benefits of an $\mathrm{Rx}$-to-OTC switch. The case of over-the-counter $\mathrm{H}_{2}$ blockers. Med Care 1990;28:834-52

17. Carr PL, Felsenstein D, Friedman RH. Evaluation and management of vaginitis. J Gen Intern Med 1998;13:335-46. 